

\section{DISCLAIMER}

This report was prepared as an account of work sponsored by an agency of the United States Government. Neither the United States Government nor any agency Thereof, nor any of their employees, makes any warranty, express or implied, or assumes any legal liability or responsibility for the accuracy, completeness, or usefulness of any information, apparatus, product, or process disclosed, or represents that its use would not infringe privately owned rights. Reference herein to any specific commercial product, process, or service by trade name, trademark, manufacturer, or otherwise does not necessarily constitute or imply its endorsement, recommendation, or favoring by the United States Government or any agency thereof. The views and opinions of authors expressed herein do not necessarily state or reflect those of the United States Government or any agency thereof. 


\section{DISCLAIMER}

Portions of this document may be illegible in electronic image products. Images are produced from the best available original document. 


\title{
Large-Aspect-Ratio Equilibrium and Stability
}

\author{
W. A. Newcomb
}

\section{Abstract}

The general guiding-center energy principle is applied to the derivation of equilibrium and stability conditions in the limiting case of a large-aspect-ratio system.

$$
\begin{aligned}
& (\underline{\nabla P})_{\perp}=\underline{Q} \underline{k}, \\
& \frac{d P}{d B}=B \frac{d}{d B}\left(\frac{Q}{B}\right),
\end{aligned}
$$

This report was prepared as an arsount of work sponsored by the United States Government. Neither Research and Development Administration Energy their employees, nor any of their contractors, subcontractors, or their employees, makes any warrenty, express or implied, or assumes any legal Liability or responsibility for the accuracy, completerness ul uxintiess un any hifimation, apparatus, product of infringe privately owned righte.

$$
P=\frac{1}{2 \mu_{0}} \cdot B^{2}+p_{1}
$$

,

$$
Q=\frac{1}{\mu_{0}} B^{2}+p_{\perp}-p_{11},
$$

$\underline{B}=$ magnetic flux density, $\mu_{0}=$ permeability of free space, $\underline{k}=$ flux-line curvature vector, $p_{1}, p_{11}=$ plasma pressure components respectively perpendicular and parallel to $B$. The derivatives $d / d B$ in (2) should be understood as directional derivatives in the parallel direction, i.e.,

$$
\frac{\mathrm{d}}{\mathrm{dB}}=(\underline{B} \cdot \underline{\nabla B})^{-1} \underline{B} \cdot \underline{\nabla} .
$$


Here and elsewhere, $|\underline{B}|$ is abbreviated as $B$. If $I$ denotes the unit vector parallel to $\underline{B}$, then

$$
\begin{aligned}
\underline{B} & =B \underline{\tau}, \\
\underline{k} & =\underline{\tau} \cdot \underline{\nabla} \underline{\tau} . \\
& =-\underline{\tau} \times(\underline{\nabla} \times \underline{\tau}) .
\end{aligned}
$$

Apply to (7) the operator $\underline{\tau} \underline{\nabla} \times$. The result reduces to

$$
\underline{\tau} \cdot \underline{\nabla} \times \underline{k}=B \underline{\tau} \cdot \underline{\nabla}(\mathbf{i} / B)
$$

where

$$
i=\underline{\tau} \cdot \underline{\nabla} \times \underline{\tau} .
$$

Physically, i represents the parallel electric current per unit magnetic flux. Now apply the same operator $\underline{\tau} \underline{\nabla} \times$ to (1). The result reduces to

$$
\underline{\tau} \cdot \underline{\nabla} \times(\underline{Q} \underline{k})=-i B \underline{\tau} \cdot \underline{\nabla}(Q / B),
$$

or if (8) is used, to

$$
\underline{\tau} \cdot \underline{k} \times \underline{\nabla Q}=B^{2} \underline{\tau} \cdot \underline{\nabla}\left(Q i / B^{2}\right) .
$$

Let us define

$$
H=d P / d B \text {, }
$$

or

$$
H=B \frac{d}{d B}\left(\frac{Q}{B}\right) \text {, }
$$

if (2) is used. The necessary and sufficient conditions for local stability at any given point are

$$
\mathrm{H}>\mathrm{O}
$$


(for stability against the mirror mode), and

$$
Q>0
$$

(for stability against the firehose mode). These are also the necessary and sufficient conditions for well-posedness of the equilibrium problem. We require them to be satisfied everywhere.

We now introduce flux coordinates $\psi, \theta$ with the property that

$$
\begin{aligned}
& \underline{B}=\underline{\nabla} \psi \times \underline{\nabla \theta}, \\
& \underline{B} \cdot \underline{\nabla} \psi=\underline{B} \cdot \underline{\nabla \theta}=0 .
\end{aligned}
$$

The pressure distribution is assumed to be of the special form

$$
\begin{aligned}
& p_{11}=p_{11}(\psi, B), \\
& p_{\perp}=p_{1}(\psi, B),
\end{aligned}
$$

i.e., independent of $\theta$. The derivative $d / d B$ in (2) is actualiy the partial with respect to $B$ for fixed $\psi$. It. follows easily from (2) that

$$
p_{\perp}=B \frac{d}{d B}\left(\frac{p_{11}}{B}\right)
$$

so that only $p_{11}(\psi, B)$ need be given.

Henceforth we assume a large-aspect-ratio system. Lengths in the $x, y$ plane are small (of order $\lambda$, say) compared with the axial scale length (in the $z$ direction), and the field components $B_{x}, B_{y}$ are small, of order $\lambda$, compared with $B_{z}$. Also, $B_{z}$ vanishes nowhere. In all calculations henceforth, all terms of relative order $\lambda^{2}$ are systematically dropped. Take the unit of length to be on the order of the transverse scale length. All gradients in the $z$ direction are then of order $\lambda$. The curvature vector $\underline{k}$ is of order $\lambda^{2}$. If $d \ell$ 
denotes the element of arc, length a long a flux line (constant $\psi, \theta$ ), then

$$
\mathrm{d} l=\mathrm{d} z \text {. }
$$

Furthermore

$$
B_{z}=B
$$

Here as elsewhere, all terms of order $\lambda^{2}$ are dropped.

From (1) to lowest order in $\lambda^{2}$ follows $(\underline{\nabla} P)_{1}=0$, or

$$
P .=P(z) \text {. }
$$

Furthermore

$$
P=\frac{1}{2 \mu_{0}} B_{v a c}^{2}
$$

where. $\underline{B}_{v a c}$ denotes the vacuum field. (Presumably $\underline{B}_{v a c}$ is given, to lowest order in $\lambda^{2}$, as a function only of $z$. Any terms depending on $x$ and $y$ are relatively of order $\lambda^{2}$.) Given $P(z)$ and also $p_{11}(\psi, B)$, it requires only a functional inversion to determine $B, p_{11}, p_{1}$, and $Q$ as functions of $\psi$ and $z$. All of these are independent of $\theta$.

A complete description of the equilibrium requires also a determination of the coordinate transformation from $(\psi, \theta, z)$ to $(x, y, z)$. It is thus required to determine the unknown functions $x(\psi, \theta, z)$ and $y(\psi, \theta, z)$, or in short, to determine the flux-line geometry.

One equation in the two unknowns $x, y$ is immediately obtainable from the definition of $\psi$ and $\theta$ as flux coordinates:

$$
\frac{\partial(x, y)}{\partial(\psi, \theta)}=\frac{1}{B}
$$


where $B$ is known as a function of $\psi$ and $z$. (The usual notation is used here to denote the Jacobian of $x$ and $y$ with respect to $\psi$ and $\theta_{\text {.) }}$ To get a second relation, we first define the covariant curvature components 9 , th so that

$$
\underline{k}=\underline{9} \underline{\nabla} \dot{\psi}+\underline{\underline{\nabla}} \underline{\theta} .
$$

If primes are used to denote partial derivatives with respect to $z$ for fixed $\psi, \theta$, then

$$
\begin{aligned}
& \text { Я }=x^{\prime \prime} \frac{\partial x}{\partial \psi}+y^{\prime \prime} \frac{\partial y}{\partial \psi} . \\
& H=x^{\prime \prime} \frac{\partial x}{\partial \theta}+y^{\prime \prime} \frac{\partial y}{\partial \theta} .
\end{aligned}
$$

Furthermore the definition (9) reduces to

$$
i=B\left[\frac{\partial\left(x^{\prime}, x\right)}{\partial(\psi, \theta)}+\frac{\partial\left(y^{\prime}, y\right)}{\partial(\psi, \theta)}\right] \text {. }
$$

Then finally, from (11), we get the desired second equation in $x$ and $y$ in the form of

$$
10 \frac{\partial Q}{\partial \psi}=-B\left(\frac{Q i}{B^{2}}\right)^{\prime}
$$

wherein $Q$ is known as a function of $\psi$ and $z$. (The partial $\partial Q / \partial \psi$ is taken for fixed z.) Presumably (25) and (30), in conjunction with appropriate boundary conditions, would suffice for the determination of the unknown coordinates $x(\psi, \theta, z)$ and $y(\psi, \theta, z)$, at least in principle.

Now let us assume that a large-aspect-ratio equilibrium solution has somehow been found, so that $x$ and $y$, together with all partial derivatives appearing in (27), (28), and (29), are known. We go on to the question of 
stability. The analysis here will be based on the standard guiding-center energy principle. It is taken as the criterion of stability that $w$, the guiding-center energy integral, be positive for every admissible choice of the displacement vector $\underline{\xi}$. The energy integral may be given as

$$
\begin{aligned}
W & =\frac{1}{2} \int\left[H(\underline{\nabla} \cdot \underline{\xi}+\underline{k} \cdot \underline{\xi})^{2}+\underline{\xi} \cdot \underline{\nabla Q} \underline{\xi} \cdot \underline{k}\right. \\
& +Q\left(\delta \underline{\tau} \cdot \delta \underline{\tau}+(\underline{k} \cdot \underline{\xi})^{2}+2 \underline{k} \cdot \underline{\xi} \underline{\nabla} \cdot \underline{\xi}\right. \\
& +i \underline{\tau} \cdot \underline{\xi} \times \delta \underline{\tau})] \mathrm{dV},
\end{aligned}
$$

plus unimportant terms, where

$$
\delta \underline{\tau}=B^{-1}[\underline{\nabla} \times(\underline{\xi} \times \underline{B})]_{\perp},
$$

and where $\underline{\xi}$ is restricted to satisfy

$$
\underline{\xi} \cdot \underline{\tau}=0
$$

The terms regarded as "unimportant". consist of a vacuum contribution $W_{\text {vac }}$ (from the vâcuum region exterior to the lateral plasma boundary) and a socalled "orbital" contribution Worb involving complicated averages over the unperturbed particle trajectories. Both these terms are nonnegative in all cases (hence never destabilizing) and of higher order in $\lambda^{2}$ in all marginal cases (hence never stabilizing either, under the conditions of interest here).

If all higher-order terms are dropped, then

$$
W=\frac{1}{2} \int H(\underline{\nabla} \cdot \underline{\xi})^{2} d V,
$$

and this is always positive as a result of the local stability condition (14) 
(which we assume to be satisfied). It suffices, therefore, to restrict attention to incompressible displacements in lowest order. That is to say, $\underline{\nabla} \cdot \underline{\xi}=0\left(\lambda^{2}\right)$. Then, to lowest nonvanishing order,

$$
W=\frac{1}{2} \int(\underline{\xi} \cdot \underline{\nabla Q} \underline{\underline{\xi}} \cdot \underline{k}+Q \underline{Q} \underline{\tau} \cdot \delta \underline{\tau}+i \underline{Q} \underline{\underline{\tau}} \cdot \underline{\xi} \times \delta \underline{\tau}) d V .
$$

At this point, we can also. drop the $0\left(\lambda^{2}\right)$ term in the side condition and rewrite it simply as

$$
\underline{\nabla} \cdot \underline{\xi}=0
$$

Now let us represent the $\underline{\xi}$ vector by its contravariant components

$$
X=\underline{\xi} \cdot \underline{\nabla} \psi, \quad Y=\underline{\xi} \cdot \underline{\nabla} \theta .
$$

In terms of $X$ and. $Y$,

$$
\begin{gathered}
\underline{\xi} \cdot \underline{\nabla Q}=X \frac{\partial Q}{\partial \psi}, \\
\underline{\xi} \cdot \underline{k}=A X+F Y, \\
\underline{\tau} \cdot \underline{\xi} \times \underline{\underline{\tau}}=\frac{1}{B}\left(X Y^{\prime}-X^{\prime} Y\right), \\
\delta \underline{\tau} \cdot \underline{\delta} \underline{\tau}=E X^{\prime 2}+2 F X^{\prime} Y^{\prime}+G Y^{\prime}{ }^{2} .
\end{gathered}
$$

Here the metric coefficients E, F, G are defined as follows:

$$
\begin{aligned}
& E=\left(\frac{\partial x}{\partial \psi}\right)^{2}+\left(\frac{\partial y}{\partial \psi}\right)^{2}, \\
& F=\frac{\partial x}{\partial \psi} \frac{\partial x}{\partial \theta}+\frac{\partial y}{\partial \psi} \frac{\partial y}{\partial \theta}, \\
& G=\left(\frac{\partial x}{\partial \theta}\right)^{2}+\left(\frac{\partial y}{\partial \theta}\right)^{2} .
\end{aligned}
$$


They satisfy the inequalities

$$
E>0, G>0, E G-F^{2}>0 \text {, }
$$

and it should also be noted that

$$
E G-F^{2}=\frac{1}{B^{2}}
$$

The incompressibility condition takes the form of

$$
B \frac{\partial}{\partial \psi}\left(\frac{X}{B}\right)+\frac{\partial Y}{\partial \theta}=0
$$

The volume element in (35) is

$$
d V=\frac{1}{B} d \psi d \theta d z
$$

(Here (21) has been used.) Then finally

$$
W=\int w d \psi d \theta,
$$

where

$$
\begin{aligned}
w=\frac{1}{2} \int_{0}^{L} d z\left[\frac { Q } { B } \left(E X^{\prime 2}+2 F X^{\prime} Y^{\prime}+\right.\right. & \left.+G Y^{\prime 2}\right)+\frac{\partial Q}{\partial \psi} \frac{X}{B}(G X+F Y) \\
& +\frac{i Q}{\left.B^{2}\left(X Y^{\prime}-X^{\prime} Y\right)\right] .}
\end{aligned}
$$

The ends of the system (at which B is a maximum and the plasma pressure components go to zero) are at $z=0$ and at $z=L$.

We can allow for either insulating or conducting ends (the form of the energy integral being the same in either case). At a conducting end, the boundary condition 


$$
X=Y=0
$$

is imposed as an admissibility condition on the $\underline{\xi}$ vector. If, on the other hand, the ends are insulating (this being the usual case), the $\xi$ vector can be shown to be essentially unrestricted. (It can be shown, in other words, that the physical boundary conditions in this case are such as not to have any effect on the final stability criterion.) One of the boundary conditions on the unperturbed equilibrium should also be mentioned, namely

$$
i=0
$$

(in the case of insulating ends).

Let us use (30) to eliminate the to term in w. After an integration by parts,

$$
w=\frac{1}{2} \int_{0}^{L} d z\left[\frac{Q}{B}\left(E X^{\prime 2}+2 F X^{\prime} Y^{\prime}+G Y^{\prime}{ }^{2}\right)+\frac{g}{B} \frac{\partial Q}{\partial \psi} X^{2}+\frac{2 i Q}{B^{2}} X Y^{\prime}\right] .
$$

Here use is made of either the boundary condition (49) for conducting ends or (50) for insulating ends.

We can derive a relatively simple sufficient condition for stability by dropping a positive term. First we complete the square with respect to $y$ to get

$$
w=w_{0}+\frac{1}{2} \int_{0}^{L} \frac{Q d z}{G B}\left(G Y^{\prime}+\frac{i X}{B}+F X^{\prime}\right)^{2},
$$

where $w_{0}$ depends only on $X$ and $X^{\prime}$. The additional term involving $Y^{\prime}$ is necessarily positive as a result of the local stability condition (15)(which we assume to be satisfied). (It was also noted that $G>0$. See (43).) There- 
fore $w_{0}$ is a lower bound on w. Explicitly,

$$
w \geq w_{0}
$$

where

$$
w_{0}=\frac{1}{2} \int_{0}^{L} d z\left[\frac{Q E}{B} X^{\prime 2}+\frac{g}{B} \frac{\partial Q}{\partial \psi} X^{2}-\frac{Q}{G B}\left(\frac{i X}{B}+F X^{\prime}\right)^{2}\right] .
$$

Again integrating by parts, and using whichever boundary condition, (49) or (50), is applicable, we end up with.

$$
w_{0}=\frac{1}{2} \int_{0}^{L}\left(f x^{\prime 2}+g x^{2}\right) d z
$$

where

$$
\begin{aligned}
& f=\frac{Q}{B G}\left(E G-F^{2}\right)=\frac{Q}{B^{3} G}, \\
& g=\frac{G}{B} \frac{\partial Q}{\partial \psi}+\left(\frac{i Q F}{G B^{2}}\right)^{\prime}-\frac{Q i^{2}}{G B^{3}} .
\end{aligned}
$$

Note that

$$
f>0
$$

everywhere, again as a result of (15) and (43). It is a sufficient condition for stability if, for all values of $\psi$ and $\theta$ (i.e., on every magnetic flux line), we get

$$
w_{0}>0
$$

for every admissible $X$. 
Consider now the Euler-Lagrange equation

$$
\left(f X^{\prime}\right)^{\prime}-g X=0 \text {. }
$$

(It is free of singularities as a result of (58).) We define two special solutions $x_{1}, x_{2}$ respectively satisfying the boundary conditions

$$
x_{1}=0, \quad x_{1}^{\prime}=1,
$$

and

$$
x_{2}=1, \quad x_{2}^{\prime}=0 \text {, }
$$

at $z=0$. Condition Green will be said to obtain if, for all values of $\psi$ and $\theta, X_{1}$ has no zeroes in the semiopen interval $0<z \leq L$. If the ends are conducting; Condition Green is sufficient for stability. Condition Blue will be said to obtain if Green obtains and if, in addition, for all $\psi$, $\theta$, it is found that

$$
\frac{x_{1}{ }^{\prime}}{x_{1}}>\frac{x_{2}{ }^{\prime}}{x_{2}}>0
$$

at $z=L$. If the ends are insulating, Condition Blue is sufficient for stability.

We can also derive a relatively simple necessary condition for stability by restricting attention to a special class of trial functions. Note first that from the incompressibility condition follows the existence of a scalar function $T$ such that

$$
X=B \frac{\partial T}{\partial \theta}, \quad Y=-B \frac{\partial T}{\partial \psi} .
$$


Conversely, any displacement thus derived from an arbitrarily given $T$ satisfies the requirement of incompressibility. Now let us choose a special form of $T$. Pick any arbitrary function of $\psi, \theta$, and $z$, and call this function $x_{r m s}$. We then define

$$
T=\frac{x_{r m s} \sqrt{2}}{m B} \sin m \theta \text {, }
$$

where $m$ is an arbitrarily large integer. Then

$$
\begin{aligned}
& x:=x_{r m s} \sqrt{2} \cos m \theta+\frac{\sqrt{2}}{m} \frac{\partial x_{r m s}}{\partial \theta} \sin m \theta, \\
& Y=-\frac{B \sqrt{2}}{m} \frac{\partial}{\partial \psi}\left(\frac{1}{B} x_{r m s}\right) \sin m \theta .
\end{aligned}
$$

(We also require the boundary condition $x_{r m s}=0$ in the case of conducting ends.) We now throw away all terms in the energy integral which are negligible for sufficiently large $m$, i.e., either (1) rapidly oscillating in $\theta$ or (2) small of order $\mathrm{m}^{-2}$.

The net effect of the foregoing operations is equivalent to the following substitutiuns in the integrand of $W$ :

$$
x \rightarrow x_{r m s}, \quad y \rightarrow 0,
$$

or simply $X \rightarrow X, Y \rightarrow 0$, if we agree to suppress the subscript rms. Thus the energy integral again reduces to the form of (55), namely

$$
w=\frac{1}{2} \int_{0}^{L}\left(f x^{\prime 2}+g x^{2}\right) d z,
$$


but now with

$$
\begin{aligned}
& f=Q E / B, \\
& g=\frac{A}{B} \frac{\partial Q}{\partial \psi} .
\end{aligned}
$$

Obviously it is necessary for stability that $w$ with the new values for $f$ and $g$ be positive for every admissible $x$ (where $x$ is now interpreted to mean $x_{\text {rms }}$ ).

Again we define the special Euler-Lagrange solutions $x_{1}, x_{2}$ satisfying the respective boundary conditions (61) and (62), and the conditions denoted as Green and Blue. Our final necessary condition for stability is the obvious analogue of the earlier-stated sufficient condition, namely Green for conducting ends, Blue for insulating ends.

It could happen that a given equilibrium satisfies the second version of Blue or Green (whichever applies), and at the same time fails to satisfy the first. Stability in that case remains undetermined, and a more penetrating analys is is needed. If, however, both $i$ and $F$ are identically zero (as in the case of a purely poloidal axisymmetric system), then the coefficients $f$ and $g$ in both versions of. $w$ are the same, and the question of indeterminacy does not arise. Our stability condition in this special case is both necessary and sufficient. 


\section{Internal}

A. W. Molvik

0. A. Anderson

$L-388$

T. R. Badura (File)

$L-388$

D. E. Baldwin

$L-386$

W. L: Barr

R. E. Batzel

$L-1$

H. L. Berk

D. H. Birdsal1

A. Blum

$L-386$

J. K. Boyd

$L-387$

J. A. Byers

$L-388$

J. Clauser

$L-386$

F. H. Coensgen (2) , L-382

R. H. Cohen

$L-388$

W. C. Condit

$L-386$

D. Correll

$L-387$

W. F. Cummins

$L-386$

T. A. Cutler

$L-387$

C. C. Damm

$L-386$

R. S. Devoto

$L-388$

J. Fink

$L-387$

J. H. Foote

$L-386$

T. K. Fowler (2)

$L-382$

R. Fre is

$L-388$

A. H. Futch

$L-386$

R. K. Goodman

$L-386$

C. Gormezano

$L-386$

L. S. Hall

$L-388$

G. W. Hamilton $\quad L-387$

M. A. Harrison $L-382$

C. W. Hartman $\quad \mathrm{L}-387$

W. Heckrotte L-388

J. Hiskes $\quad L-388$

J. Holdren L-386

E. B. Hooper $L-387$

R. Hornady L-386

A. L. Hunt L-386

B. M. Johnston $L-387$

T. B. Kaiser L-388

L. L. Lodestro $\mathrm{L}-387$

B. G. Logan $L-386$

N. Maron $\mathrm{L}-387$

B. McNamara L $\quad L-388$

R. H. Munger $\quad \mathrm{L}-387$

M. L. Nelson $L-382$

W. A. Newcomb $L-388$

W. E. Nexsen $L-386$

J. E. Osher L L-386

L. D. Pearlstein $L-388$

G. D. Porter $L-386$

R. F. Post L-336

D. S. Prono L-387

M. E. Rensink $L-388$

T. Rognlien $\quad L-387$

J. Sayer $\quad L-387$

J. Shearer $\quad L-387$

T. Simonen $\quad \mathrm{L}-386$

B. W. Stallard $\quad L-386$

J. J. Stewart $L-387$

K. Struve $L-387$

C. E. Taylor $\quad L-386$

W. C. Turner $\quad L-386$

D. Watson $L-388$

P. Willmann L-387

CTR Library $\quad L-387$

TID (15) L-9

W. Sharp $L-306$

TIC, Oak Ridge (27)

\section{External}

Harold Grad Harold Weitzner Courant Institute of Math. Sci. New York University

251 Mercer Street

New York, NY 10012

\section{NOTICE}

"This report was prepared as an account of work sponsored by the United States Guvernment. Neither the United States nor the United States Energy Research \& Development Administration, nor any of their employees. nor any of their contracturs, subcontractors, or their employees, makes atly warranty, express or implied, or assumes any legal liability or responsibility for the accuracy, completeness or usefulness of any information, apparatus, product or process disclosed, or represents that its use would not infringe privately-owned rights." 
